\title{
ECONOMICIDADE E DESEMPENHO PRODUTIVO DE CORDEIROS CONFINADOS SUBMETIDOS A DIETAS COM RESÍDUOS AGROINDUSTRIAIS
}

\section{ECONOMICS AND PRODUCTIVE PERFORMANCE OF FEEDLOT LAMBS FED DIETS WITH DIFFERENT AGROINDUSTRY RESIDUES}

\author{
Sérgio Carvalho ${ }^{1 *}$ \\ Cleber Cassol Pires ${ }^{1}$ \\ Tatiana Pfüller Wommer ${ }^{1}$ \\ Jusecleia Ferreira Lopes ${ }^{1}$ \\ Camila Ornelas Mônego ${ }^{1}$ \\ Veronica Milanesi Pilecco ${ }^{1}$ \\ ${ }^{1}$ Universidade Federal de Santa Maria, Santa Maria, RS, Brasil. \\ *Autor para correspondência - scarvalhoufsm@hotmail.com
}

\begin{abstract}
Resumo
Avaliou-se o aproveitamento de resíduos agroindustriais na terminação de cordeiros em sistema de confinamento. Utilizaram-se 12 cordeiros, machos, não castrados, da raça Texel, distribuídos aleatoriamente nos tratamentos compostos por diferentes resíduos incluídos na proporção de $30 \%$ da matéria seca da dieta total, sendo: casca de soja, quirera de arroz ou pó de malte de cervejaria. Os cordeiros foram confinados por 70 dias e abatidos com aproximadamente $40 \mathrm{~kg}$ de peso corporal. Para a análise econômica da alimentação fornecida, foram considerados os preços de mercado obtidos na região do estudo para os ingredientes das rações e peso vivo dos cordeiros. $\mathrm{O}$ consumo de carboidratos não fibrosos foi superior $(\mathrm{P}<0,01)$ nos cordeiros que receberam quirera de arroz. Não foi verificada diferença em relação ao consumo de matéria seca entre os tratamentos. $\mathrm{O}$ ganho de peso diário e o ganho de peso total foram superiores $(\mathrm{P}<0,05)$ nos cordeiros que receberam quirera de arroz. O lucro obtido foi superior $(\mathrm{P}<0,01)$ quando se utilizou pó de malte de cervejaria $(\mathrm{R} \$ 1,89 / \mathrm{kg}$ de ganho de peso vivo) comparado com casca de soja ( $\$$ \$ $0,96 / \mathrm{kg}$ de ganho de peso vivo) e quirera de arroz (R $\$ 1,15 /$ $\mathrm{kg}$ de ganho de peso vivo). A utilização de pó de malte de cervejaria proporciona menor custo de alimentação quando comparada com a utilização de casca de soja ou quirera de arroz, mostrando ser uma boa alternativa para terminação de cordeiros em sistema de confinamento.
\end{abstract}

Palavras-chave: consumo; ganho de peso; ovino; produção animal.

\begin{abstract}
We evaluated the use of agroindustry residues in finishing lambs in feedlot. Twelve non-castrated male Texel lambs were randomly distributed in the treatments composed of different residues at $30 \%$ of dry matter of the total diet, as follows: soybean hulls, broken rice or malt powder from a brewery. The lambs were confined for 70 days and slaughtered at approximately $40 \mathrm{~kg}$ body weight. In the economic analysis of the diet, we considered the market prices in the study region for the diet ingredients and weight of lambs. The intake of non-structural carbohydrate was higher $(\mathrm{P}<0.01)$ in lambs on the treatment with broken rice. There was no difference between treatments in dry matter consumption. Daily weight gain and total weight gain were higher $(\mathrm{P}<0.05)$ in lambs receiving broken rice. Profit was higher $(\mathrm{P}<0.01)$ when malt powder from a brewery was used ( $\mathrm{R} \$ 1.89 / \mathrm{kg}$ of live weight gain) compared with soybean hulls ( $\$ 0.96 / \mathrm{kg}$ of live weight gain) or broken rice ( $\mathrm{R} \$ 1.15 / \mathrm{kg}$ of live weight gain). The use of malt powder from a brewery provides lower cost of the diet when compared to use of soybean hulls or broken rice, proving to be a good alternative for finishing lambs in feedlot.
\end{abstract}

Keywords: animal production; intake; sheep; weight gain. 
Enviado em: 24 agosto 2012

Aceito em: 16 dezembro de 2015

\section{Introdução}

O alto potencial produtivo e o crescente consumo de carne de cordeiro são fatores que promovem estímulo para o crescimento da ovinocultura no Brasil. Entretanto, para atender essa demanda por carne de animais jovens torna-se necessário intensificar a produção e reduzir o tempo despendido para terminação dos cordeiros. Uma das formas para alcançar maiores índices produtivos seria a adoção da terminação de cordeiros em sistema de confinamento.

Contudo, um dos grandes problemas encontrados neste sistema de produção tem sido os altos custos, principalmente no que se refere à alimentação ${ }^{(1)}$. De acordo com Amaral et al. ${ }^{(2)}$, a alimentação é a base para o sucesso produtivo do sistema de confinamento, devendo-se adotar estratégias que visem aumentar a eficiência de utilização dos nutrientes, aliados à redução dos custos com alimentação. Segundo os autores, a utilização do confinamento, quando comparada a sistemas de produção a pasto, exige um capital de giro disponível superior, sendo necessário, para obtenção de êxito, melhor controle por meio da avaliação econômica do sistema produtivo.

Para se obter dietas de baixo custo, é necessário buscar por ingredientes mais baratos, como os resíduos agroindustriais. Segundo Brochier e Carvalho ${ }^{(3)}$, a utilização de resíduos agroindustriais na terminação de cordeiros em confinamento tem grande potencial, pois pode levar à redução significativa dos custos de alimentação do confinamento, além de promover a redução de impactos ambientais advindos da destinação inadequada destes resíduos, sem que ocorram perdas nos índices produtivos. Este estudo teve por objetivo realizar uma análise do consumo de nutrientes, do desempenho animal e a da viabilidade econômica do aproveitamento de diferentes resíduos agroindustriais na terminação de cordeiros em sistema de confinamento.

\section{Material e Métodos}

O experimento foi realizado numa propriedade rural, localizada no município de Novo Hamburgo, RS. Foram utilizados 12 cordeiros, machos, não-castrados, da raça Texel, nascidos de parto simples e desmamados aproximadamente aos 50 dias de idade. O período experimental foi precedido por 14 dias de adaptação à alimentação, ao manejo e às instalações.

Os cordeiros foram confinados em baias individuais, totalmente cobertas, com piso ripado e dimensão de $1,5 \mathrm{~m}^{2}$ por animal, providas de comedouros e bebedouros individuais. Os animais foram distribuídos aleatoriamente em três tratamentos, com quatro repetições cada. Os tratamentos foram constituídos por diferentes resíduos agroindustriais incluídos na proporção de $30 \%$ da matéria seca total das dietas, sendo eles: casca de soja, quirera de arroz e pó de malte de cervejaria. As dietas experimentais (Tabela 1) foram compostas por uma relação volumoso:concentrado de 40:60, com base na matéria seca (MS). O volumoso utilizado foi o feno de Tifton-85 e os concentrados eram compostos por um dos resíduos utilizados nos tratamentos, acrescido de fubá de milho, farelo de soja, calcário calcítico e sal comum. As dietas foram formuladas para serem isoprotéicas e isoenergéticas, de acordo com o $\mathrm{NRC}^{(4)}$.

$\mathrm{O}$ alimento foi fornecido ad libitum, duas vezes ao dia, sendo os horários de arraçoamento às 7:30 e 17:30 horas. A quantidade fornecida foi ajustada diariamente de modo a manter as sobras em 15\% do total fornecido, de modo a garantir o consumo voluntário máximo dos animais. Os alimentos e as sobras foram pesados diariamente a fim de se estimar o consumo de nutrientes dos cordeiros. Diariamente, foram coletadas amostras dos alimentos e das sobras, sendo que, no final do período experimental, foram feitas amostras compostas das mesmas. As amostras foram devidamente identificadas e armazenadas em freezer à temperatura de $-20^{\circ} \mathrm{C}$ para posteriores análises laboratoriais. Todas as amostras foram pré-secas em estufa ventilada a $65^{\circ} \mathrm{C}$ e, posteriormente, trituradas em moinho tipo 
Willey com peneira de $1 \mathrm{~mm}$. Foram determinadas (Tabela 2) as concentrações de matéria seca (MS), matéria orgânica $(\mathrm{MO})$, proteína bruta $(\mathrm{PB})$, extrato etéreo (EE), fibra em detergente neutro (FDN) e cinzas $(\mathrm{CIN})$, segundo Silva e Queiroz ${ }^{(5)}$. Os carboidratos totais (CHT) dos alimentos fornecidos e das sobras foram calculados segundo Carvalho et al. ${ }^{(6)}$, em que CHT $(\%)=100-(\% \mathrm{~PB}+\% \mathrm{EE}$ $+\% \mathrm{CIN}$ ), e os carboidratos não-fibrosos (CNF) pela diferença de CHT - FDN. Todas as análises laboratoriais foram realizadas na Central Analítica do Centro Universitário Feevale.

Os cordeiros foram pesados no início e final da fase experimental, bem como a cada 14 dias, após jejum de 12 horas, sendo abatidos quando se atingiu 70 dias de período experimental.

Tabela 1: Proporções dos ingredientes (\% MS) e composição bromatológica das dietas experimentais

\begin{tabular}{|c|c|c|c|}
\hline & \multicolumn{3}{|c|}{ Tratamento } \\
\hline & Casca de soja & Quirera de arroz & $\begin{array}{c}\text { Pó de malte de } \\
\text { cervejaria }\end{array}$ \\
\hline \multicolumn{4}{|c|}{ Proporções dos ingredientes (\% MS) } \\
\hline Feno Tifton-85 & 40,00 & 40,00 & 40,00 \\
\hline Fubá de milho & 18,41 & 12,46 & 13,19 \\
\hline Farelo de soja & 10,10 & 15,74 & 15,02 \\
\hline Casca de soja & 30,00 & ---- & ---- \\
\hline Quirera de arroz & $-\cdots$ & 30,00 & ---- \\
\hline $\begin{array}{l}\text { Pó de malte de } \\
\text { cervejaria }\end{array}$ & --.- & ---- & 30,00 \\
\hline Calcário calcítico & 0,49 & 0,80 & 0,79 \\
\hline Sal & 1,00 & 1,00 & 1,00 \\
\hline \multicolumn{4}{|c|}{ Composição bromatológica (\% da MS) } \\
\hline MS & 85,25 & 85,02 & 84,45 \\
\hline MO & 92,89 & 93,26 & 92,74 \\
\hline PB & 16,00 & 16,00 & 16,00 \\
\hline $\mathrm{EE}$ & 1,91 & 1,36 & 1,48 \\
\hline FDN & 48,69 & 41,02 & 48,93 \\
\hline $\mathrm{CHT}$ & 74,98 & 75,90 & 75,26 \\
\hline $\mathrm{CNF}$ & 26,29 & 34,89 & 26,33 \\
\hline CIN & 5,62 & 4,94 & 5,47 \\
\hline $\mathrm{EL}(\mathrm{Mcal} / \mathrm{kg})$ & 1,48 & 1,54 & 1,50 \\
\hline $\mathrm{Ca}$ & 0,50 & 0,50 & 0,50 \\
\hline $\mathrm{P}$ & 0,24 & 0,28 & 0,33 \\
\hline
\end{tabular}

$\mathrm{MS}=$ matéria seca; $\mathrm{MO}=$ matéria orgânica; $\mathrm{PB}=$ proteína bruta; $\mathrm{EE}=$ extrato etéreo; FDN $=$ fibra em detergente neutro; $\mathrm{CHT}=$ carboidratos totais; $\mathrm{CNF}=$ carboidratos não-fibrosos; $\mathrm{CIN}=$ cinzas; $\mathrm{EL}=$ energia líquida; $\mathrm{Ca}=$ cálcio; $\mathrm{P}$ $=$ fósforo.

A análise econômica foi realizada somente em relação à alimentação oferecida para os animais, não sendo considerados os demais custos do sistema (mão de obra, instalações, água, energia etc.). Para se calcular a rentabilidade da alimentação oferecida no experimento, foram considerados os preços de mercado obtidos na região do estudo para os ingredientes das rações e peso vivo dos cordeiros. Consideraram-se os seguintes valores: $\mathrm{R} \$ 3,50 / \mathrm{kg}$ de peso vivo do cordeiro gordo, $\mathrm{R} \$ 0,40 / \mathrm{kg}$ de feno de Tifton-85, R\$ 0,55/kg de milho moído, R $\$ 0,85 / \mathrm{kg}$ de farelo de soja, $\mathrm{R} \$ 0,35 / \mathrm{kg}$ de casca de soja, $\mathrm{R} \$ 0,34 / \mathrm{kg}$ de quirera de arroz, $\mathrm{R} \$ 0,12 / \mathrm{kg}$ de pó de malte de cervejaria, $\mathrm{R} \$ 0,02 / \mathrm{kg}$ de calcário calcítico e R\$ 0,42/kg de sal. 
De posse do custo de cada ração e do seu consumo médio individual ao longo do experimento, foi calculado o resultado econômico proporcionado por ração, utilizando-se as seguintes equações:

Custo diário de feno $(\mathrm{R} \$ /$ animal/dia $)=$ fornecido de matéria natural de feno $(\mathrm{kg} / \mathrm{dia}) \mathrm{x}$ custo do feno $(\mathrm{R} \$ / \mathrm{kg})$

Custo diário do concentrado $(\mathrm{R} \$ /$ animal $/$ dia $)$ = fornecido de matéria natural do concentrado $(\mathrm{kg} / \mathrm{dia})$ $\mathrm{x}$ custo do concentrado $(\mathrm{R} \$ / \mathrm{kg})$;

Custo diário total $(\mathrm{R} \$ /$ animal $/$ dia $)=$ custo diário de feno $(\mathrm{R} \$ /$ animal $/$ dia $)+$ custo diário de concentrado (R\$/animal/dia);

Custo total de produção $(\mathrm{R} \$ /$ animal $)=$ custo diário total $(\mathrm{R} \$ /$ animal/dia $)$ x 70 (número de dias do confinamento);

Receita do peso vivo $(\mathrm{R} \$$ /animal $)$ = peso vivo ao abate de cada cordeiro $(\mathrm{kg}) \times$ preço pago por $\mathrm{kg}$ de peso vivo do cordeiro $(\mathrm{R} \$ \mathrm{~kg})$;

Rentabilidade do peso vivo $(\mathrm{R} \$ /$ animal $)=$ receita do peso vivo $(\mathrm{R} \$ /$ animal $)$ - custo total de produção (R\$/animal);

Receita do ganho de peso vivo $(\mathrm{R} \$ /$ animal $)=$ ganho de peso total obtido por cada cordeiro no confinamento $(\mathrm{kg})$ x preço pago por $\mathrm{kg}$ de peso vivo do cordeiro $(\mathrm{R} \$ / \mathrm{kg})$;

Rentabilidade do ganho de peso vivo $(\mathrm{R} \$ /$ animal $)$ = receita do ganho de peso vivo (R \$/animal) custo total de produção (R \$/animal);

Rentabilidade obtida por $\mathrm{kg}$ de ganho de peso vivo $(\mathrm{R} \$ / \mathrm{kg})$ = lucro do ganho de peso vivo (R\$/ animal) / ganho de peso total obtido por cada cordeiro no confinamento (kg);

Rentabilidade diária obtida por animal $(\mathrm{R} \$ / \mathrm{dia})=$ lucro por $\mathrm{kg}$ de ganho de peso vivo $(\mathrm{R} \$ / \mathrm{kg})$ - custo diário total (R\$/animal/dia).

O delineamento experimental utilizado foi inteiramente casualizado com três tratamentos e quatro repetições, sendo cada cordeiro considerado uma unidade experimental. Os dados foram analisados utilizando-se o pacote estatístico SAS ${ }^{(7)}$, sendo submetidos à análise de variância e ao teste de Tukey ao nível de 5\% de probabilidade.

Tabela 2: Teores médios de matéria seca (MS), matéria orgânica (MO), proteína bruta (PB), extrato etéreo (EE), fibra em detergente neutro (FDN), carboidratos totais ( $\mathrm{CHT})$, carboidratos não-fibrosos $(\mathrm{CNF})$, cinzas $(\mathrm{CIN})$, energia líquida $(\mathrm{EL})$, cálcio $(\mathrm{Ca})$ e fósforo $(\mathrm{P})$, dos ingredientes utilizados na formulação das dietas experimentais

\begin{tabular}{lccccccc}
\hline Item (\%) & $\begin{array}{c}\text { Feno } \\
\text { Tifton-85 }\end{array}$ & $\begin{array}{c}\text { Fubá } \\
\text { de milho }\end{array}$ & $\begin{array}{c}\text { Farelo } \\
\text { de soja }\end{array}$ & $\begin{array}{c}\text { Casca de } \\
\text { soja }\end{array}$ & $\begin{array}{c}\text { Quirera } \\
\text { de arroz }\end{array}$ & $\begin{array}{c}\text { Pó de } \\
\text { malte de } \\
\text { cervejaria }\end{array}$ & $\begin{array}{c}\text { Calcário } \\
\text { Calcítico }\end{array}$ \\
\hline MS & 85,49 & 84,80 & 82,70 & 85,34 & 84,79 & 82,85 & 100 \\
MO & 92,22 & 98,59 & 91,88 & 95,22 & 98,73 & 96,79 & ----- \\
PB & 10,92 & 10,65 & 44,11 & 17,39 & 11,21 & 12,01 & ---- \\
EE & 1,01 & 3,90 & 1,32 & 2,17 & 0,85 & 1,20 & ---- \\
FDN & 78,20 & 15,82 & 25,26 & 40,03 & 12,84 & 39,44 & ---- \\
CHT & 80,29 & 84,04 & 46,45 & 75,66 & 86,67 & 83,58 & ---- \\
CNF & 2,09 & 68,22 & 21,19 & 35,63 & 73,83 & 44,14 & $-\cdots$ \\
CIN & 7,78 & 1,41 & 8,12 & 4,78 & 1,27 & 3,21 & $-\cdots--$ \\
EL (Mcal/kg) & 1,101 & 1,961 & 1,941 & $1,60^{2}$ & 1,842 & 1,702 & ----- \\
Ca & 0,42 & 0,03 & 0,30 & 0,44 & 0,03 & 0,05 & 34 \\
P & 0,19 & 0,30 & 0,68 & 0,14 & 0,21 & 0,38 & 0,02 \\
\hline
\end{tabular}

1 - Valor obtido em Carvalho et al. (6).

2 - Valor obtido no NRC (4). 


\section{Resultados e Discussão}

O consumo de carboidratos não-fibrosos, expresso em quilograma por dia ( $\mathrm{kg} / \mathrm{dia})$, porcentagem do peso vivo (\%PV) e gramas por unidade de tamanho metabólico $\left(\mathrm{g} / \mathrm{kg}^{0,75}\right)$ (Tabelas 3, 4 e 5), foi superior $(\mathrm{P}<0,01)$ nos cordeiros do tratamento com quirera de arroz quando comparados aos cordeiros dos tratamentos com casca de soja ou pó de malte de cervejaria. Esse resultado pode ser explicado pela maior concentração de carboidratos não-fibrosos encontrados na quirera de arroz quando comparada aos outros resíduos utilizados (Tabela 2). Verificou-se também que o consumo de extrato etéreo foi superior $(\mathrm{P}<0,01)$, quando expresso em $\mathrm{kg} / \mathrm{dia}$ e $\mathrm{g} / \mathrm{kg}^{0,75}$, nos cordeiros do tratamento com casca de soja em relação aos cordeiros dos tratamentos com quirera de arroz ou pó de malte de cervejaria, o que também pode ser atribuído à sua maior concentração no tratamento da casca de soja em relação aos tratamentos dos outros resíduos utilizados.

Tabela 3: Valores médios para os consumos de matéria seca (CMS), matéria orgânica (CMO), proteína bruta (CPB), extrato etéreo (CEE), fibra em detergente neutro (CFDN), carboidratos totais (CCHT) e carboidratos não-fibrosos estruturais (CCNF), em kg/dia, e de energia líquida (CEL), em $\mathrm{Mcal} / \mathrm{dia}$, de acordo com os tratamentos

\begin{tabular}{lccccc}
\hline \multirow{2}{*}{ Variáveis } & \multicolumn{3}{c}{ Tratamento } & Média & Pr>F \\
\cline { 2 - 4 } & Casca de soja & $\begin{array}{c}\text { Quirera de } \\
\text { arroz }\end{array}$ & $\begin{array}{c}\text { Pó de malte de } \\
\text { cervejaria }\end{array}$ & & \\
\hline CMS & $1,070 \pm 0,17$ & $1,184 \pm 0,19$ & $1,003 \pm 0,10$ & 1,086 & 0,3200 \\
CMO & $0,970 \pm 0,16$ & $1,078 \pm 0,18$ & $0,900 \pm 0,09$ & 0,982 & 0,2872 \\
CPB & $0,171 \pm 0,03$ & $0,188 \pm 0,03$ & $0,16 \pm 0,016$ & 0,173 & 0,3262 \\
CEE & $0,019 \pm 0,003^{\mathrm{a}}$ & $0,015 \pm 0,002^{\mathrm{b}}$ & $0,014 \pm 0,001^{\mathrm{b}}$ & 0,016 & 0,0376 \\
$\mathrm{CFDN}$ & $0,523 \pm 0,08$ & $0,49 \pm 0,08$ & $0,494 \pm 0,05$ & 0,503 & 0,7865 \\
$\mathrm{CCHT}$ & $0,783 \pm 0,13$ & $0,878 \pm 0,14$ & $0,731 \pm 0,07$ & 0,797 & 0,2725 \\
$\mathrm{CCNF}$ & $0,272 \pm 0,04^{\mathrm{b}}$ & $0,399 \pm 0,066^{\mathrm{a}}$ & $0,252 \pm 0,03^{\mathrm{b}}$ & 0,308 & 0,0043 \\
$\mathrm{CEL}$ & $1,580 \pm 0,25$ & $1,820 \pm 0,30$ & $1,500 \pm 0,16$ & 1,630 & 0,2028 \\
\hline
\end{tabular}

Médias seguidas de letras diferentes na linha diferem $(\mathrm{P}<0,05)$ pelo teste Tukey.

Tabela 4: Valores médios para os consumos de matéria seca (CMS), matéria orgânica (CMO), proteína bruta (CPB), extrato etéreo (CEE), fibra em detergente neutro (CFDN), carboidratos totais (CCHT) e carboidratos não-fibrosos (CCNF), em \% PV, e de energia líquida (CEL), em Mcal $/ \mathrm{kg}$ $\mathrm{PV}$, de acordo com os tratamentos

\begin{tabular}{lccccc}
\hline \multirow{2}{*}{ Variáveis } & \multicolumn{3}{c}{ Tratamento } & Média & Pr>F \\
\cline { 2 - 4 } & Casca de soja & $\begin{array}{c}\text { Quirera de } \\
\text { arroz }\end{array}$ & $\begin{array}{c}\text { Pó de malte de } \\
\text { cervejaria }\end{array}$ & & \\
\hline CMS & $3,37 \pm 0,38$ & $3,50 \pm 0,41$ & $3,34 \pm 0,13$ & 3,40 & 0,7581 \\
CMO & $3,05 \pm 0,36$ & $3,19 \pm 0,38$ & $3,00 \pm 0,11$ & 3,08 & 0,6860 \\
CPB & $0,54 \pm 0,06$ & $0,56 \pm 0,06$ & $0,53 \pm 0,02$ & 0,54 & 0,7860 \\
CEE & $0,06 \pm 0,008^{\mathrm{a}}$ & $0,04 \pm 0,005^{\mathrm{b}}$ & $0,05 \pm 0,007^{\mathrm{ab}}$ & 0,05 & 0,0050 \\
$\mathrm{CFDN}$ & $1,65 \pm 0,19$ & $1,45 \pm 0,16$ & $1,65 \pm 0,06$ & 1,58 & 0,1546 \\
$\mathrm{CCHT}$ & $2,46 \pm 0,29$ & $2,60 \pm 0,31$ & $2,43 \pm 0,09$ & 2,50 & 0,6382 \\
$\mathrm{CCNF}$ & $0,86 \pm 0,10^{\mathrm{b}}$ & $1,18 \pm 0,14^{\mathrm{a}}$ & $0,84 \pm 0,03^{\mathrm{b}}$ & 0,96 & 0,0018 \\
$\mathrm{CEL}$ & $0,05 \pm 0,008$ & $0,05 \pm 0,005$ & $0,05 \pm 0,006$ & 0,05 & 0,7674 \\
\hline
\end{tabular}

Médias seguidas de letras diferentes na linha diferem $(\mathrm{P}<0,05)$ pelo teste Tukey. 
Tabela 5: Valores médios para os consumos de matéria seca (CMS), matéria orgânica (CMO), proteína bruta $(\mathrm{CPB})$, extrato etéreo (CEE), fibra em detergente neutro (CFDN), carboidratos totais (CCHT) e carboidratos não-fibrosos (CCNF), em g/ $\mathrm{kg}^{0,75}$, e de energia líquida (EL), em $\mathrm{Mcal} / \mathrm{kg}^{0,75}$, de acordo com os tratamentos

\begin{tabular}{|c|c|c|c|c|c|}
\hline \multirow[b]{2}{*}{ Variáveis } & \multicolumn{3}{|c|}{ Tratamento } & \multirow[b]{2}{*}{ Média } & \multirow[b]{2}{*}{$\operatorname{Pr}>\mathbf{F}$} \\
\hline & Casca de soja & Quirera de arroz & $\begin{array}{c}\text { Pó de malte de } \\
\text { cervejaria }\end{array}$ & & \\
\hline CMS & $79,75 \pm 9,33$ & $84,09 \pm 9,88$ & $77,91 \pm 3,11$ & 80,58 & 0,5585 \\
\hline $\mathrm{CMO}$ & $72,19 \pm 8,83$ & $76,54 \pm 9,19$ & $69,96 \pm 2,86$ & 72,90 & 0,4837 \\
\hline $\mathrm{CPB}$ & $12,73 \pm 1,49$ & $13,41 \pm 1,58$ & $12,41 \pm 0,49$ & 12,85 & 0,5552 \\
\hline CEE & $1,48 \pm 0,18^{\mathrm{a}}$ & $1,11 \pm 0,13^{b}$ & $1,11 \pm 0,05^{\mathrm{b}}$ & 1,23 & 0,0042 \\
\hline CFDN & $39,00 \pm 4,55$ & $34,82 \pm 4,06$ & $38,44 \pm 1,52$ & 37,42 & 0,2607 \\
\hline $\mathrm{CCHT}$ & $58,30 \pm 7,13$ & $62,34 \pm 7,48$ & $56,83 \pm 2,32$ & 59,16 & 0,4506 \\
\hline $\mathrm{CCNF}$ & $20,29 \pm 2,49^{b}$ & $28,34 \pm 3,43^{\mathrm{a}}$ & $19,59 \pm 0,81^{b}$ & 22,74 & 0,0013 \\
\hline CEL & $0,11 \pm 0,01$ & $0,13 \pm 0,01$ & $0,11 \pm 0,01$ & 0,12 & 0,1613 \\
\hline
\end{tabular}

Médias seguidas de letras diferentes na linha diferem $(\mathrm{P}<0,05)$ pelo teste Tukey.

Não foi verificada diferença significativa $(P>0,05)$ entre os tratamentos em relação ao consumo de matéria seca. Isto pode ser explicado pelo fato de as dietas apresentarem uma similaridade entre os teores de fibra em detergente neutro (todas acima de 40\% de FDN, embora com uma pequena inferioridade no tratamento da quirera de arroz) e de energia. De acordo com Fontenele et al. ${ }^{(8)}$, quando o teor de FDN é baixo e a densidade energética da ração é alta, o consumo é limitado pela demanda energética, não ocorrendo repleção ruminal. Por outro lado, para rações com densidade energética baixa (alto teor de FDN), o consumo será limitado por limitação física ou enchimento do rúmen-retículo ${ }^{\left({ }^{9}\right)}$, reduzindo o desempenho animal. Como as dietas utilizadas no presente estudo apresentavam uma similaridade entre os teores de fibra em detergente neutro e energia, os resultados para o consumo de matéria seca também foram similares. Como consequência, os consumos de matéria orgânica, proteína bruta, carboidratos totais e fibra em detergente neutro também não diferiram $(\mathrm{P}>0,05)$ entre os tratamentos. $\mathrm{O}$ consumo de matéria seca está de acordo com a recomendação do $\mathrm{NRC}^{(4)}$ para ovinos desta categoria, os quais variam de 1 a 1,3 kg de MS/animal/dia. Com relação ao consumo de matéria seca em \%PV e $\mathrm{g} / \mathrm{kg}^{0,75}$, os resultados obtidos estão de acordo com Cardoso et al. ${ }^{(10)}$, que avaliaram o consumo de nutrientes e o desempenho de cordeiros alimentados em confinamento com dietas contendo diferentes níveis de fibra em detergente neutro e observaram valores médios de 3,46\% e 76,75 g/ $/ \mathrm{kg}^{0,75}$. Já em relação ao consumo de matéria orgânica, expresso em $\mathrm{kg} / \mathrm{dia}$, o valor médio do presente experimento é superior ao observado por Kozloski et al. ${ }^{(11)}$, que testaram diferentes níveis de fibra em detergente neutro na dieta de cordeiros mestiços Texel, Corriedale e Ile de France, com peso vivo médio de $35 \mathrm{~kg}$, e observaram um consumo médio de $0,826 \mathrm{~kg} /$ dia. Os autores supracitados verificaram um consumo médio para FDN de 0,255 kg/dia, valor esse 49,3\% inferior ao do presente estudo, o que pode ser explicado pela menor proporção de FDN presente nas dietas utilizadas por aqueles autores. Por outro lado, o valor médio observado para o consumo de FDN de $0,50 \mathrm{~kg} /$ dia está de acordo com o verificado por Alves et al. ${ }^{(12)}$, os quais testaram diferentes níveis de energia em dietas para ovinos da raça Santa Inês e observaram um consumo médio de $0,47 \mathrm{~kg}$ de FDN/dia, em uma dieta contendo $58,3 \%$ de FDN. Baseado nas comparações acima, pode-se afirmar que alimentos com elevado teor de FDN poderão ter sua ingestão restringida e, provavelmente, proporcionar limitação da expressão do potencial genético do animal para produção ${ }^{(13)}$. Entretanto, no presente estudo, de acordo com os resultados observados e comparados com a literatura, não foram evidenciadas possíveis restrições no consumo de matéria seca, o que permite sugerir que não ocorreu regulação física do consumo dos animais.

O ganho de peso diário e o ganho de peso total foram superiores $(\mathrm{P}<0,05)$ nos cordeiros do tratamento 
com quirera de arroz em relação aos cordeiros do tratamento com pó de malte de cervejaria (Tabela 6), porém não diferiram $(\mathrm{P}>0,05)$ em relação àqueles do tratamento com casca de soja. Esse resultado pode ser explicado a partir da afirmativa de Vieira et al. ${ }^{(14)}$ de que a menor proporção de carboidratos fibrosos e o maior teor de carboidratos não fibrosos nas dietas levam ao aumento da digestibilidade da MS e da MO, aspecto esse que proporciona uma maior disponibilidade de nutrientes para o metabolismo dos animais, havendo incremento no ganho de peso.

Com relação ao ganho de peso, deve-se enfatizar que desse depende o tempo necessário para que um cordeiro atinja a condição de abate, sendo que, quanto mais rapidamente essa condição for atingida, maior será a sua contribuição para o processo de produção de carne. Nesse sentido, o valor médio observado para o ganho de peso diário dos cordeiros, independente do tratamento, pode ser considerado satisfatório (média de $260 \mathrm{~g} / \mathrm{dia}$ ), o que fica caracterizado quando comparamos esse resultado com aquele obtido por Carvalho et al. ${ }^{(15)}$, que quais verificaram um valor médio de 0,171 $\mathrm{kg} /$ dia em cordeiros da raça Texel, desmamados com 60 dias de idade e confinados por um período de 84 dias, recebendo uma dieta contendo feno de Tifton-85 e concentrado na proporção 40:60 na matéria seca.

Tabela 6: Valores médios para peso vivo inicial (PI), peso vivo ao abate (PVA), ganho de peso médio diário (GMD), ganho de peso total (GT) e conversão alimentar (CA), de acordo com os tratamentos

\begin{tabular}{lccccc}
\hline & \multicolumn{3}{c}{ Tratamento } & & \\
\cline { 2 - 4 } Variáveis & Casca de soja & $\begin{array}{c}\text { Quirera de } \\
\text { arroz }\end{array}$ & $\begin{array}{c}\text { Pó de malte } \\
\text { de cervejaria }\end{array}$ & Média & Pr>F \\
\hline PI (kg) & $22,61 \pm 3,06$ & $22,04 \pm 3,58$ & $22,57 \pm 2,91$ & 22,41 & 0,9611 \\
PVA (kg) & $40,89 \pm 3,91$ & $44,16 \pm 4,67$ & $36,90 \pm 3,93$ & 40,65 & 0,0994 \\
GMD (kg/dia) & $0,26 \pm 0,05^{\mathrm{ab}}$ & $0,32 \pm 0,03^{\mathrm{a}}$ & $0,21 \pm 0,03^{\mathrm{b}}$ & 0,26 & 0,0106 \\
GT (kg) & $18,28 \pm 3,77^{\mathrm{ab}}$ & $22,12 \pm 2,23^{\mathrm{a}}$ & $14,33 \pm 2,01^{\mathrm{b}}$ & 18,24 & 0,0107 \\
CA & $4,14 \pm 0,50$ & $3,94 \pm 0,72$ & $5,27 \pm 1,03$ & 4,45 & 0,0806 \\
\hline
\end{tabular}

Médias seguidas de letras diferentes na linha diferem $(\mathrm{P}<0,05)$ pelo teste Tukey.

Com relação à conversão alimentar, observou-se que a mesma não diferiu $(\mathrm{P}>0,05)$ entre os tratamentos. Segundo Pilar et al. ${ }^{(16)}$, a conversão alimentar é reduzida à medida que o peso vivo aumenta, proporcionada pela elevação do consumo de matéria seca em relação ao ganho de peso dos animais. Embora diferenças no ganho de peso diário tenham sido observadas, elas não foram suficientes para proporcionar diferenças significativas nas conversões alimentares entre os tratamentos. Esta semelhança determinada nas conversões alimentares também pode ser explicada pela similaridade observada no consumo de matéria seca dos cordeiros. O valor médio obtido de 4,45:1 pode ser considerado compatível com a idade dos animais, com a raça e com o sistema de terminação utilizado, sendo similar aos valores relatados por Cardoso et al. ${ }^{(10)}$, que verificaram um valor médio de 4,21:1 em cordeiros cruza Ile de France x Texel, confinados e recebendo uma dieta contendo $43 \%$ de fibra em detergente neutro, e abatidos aos 112 dias de idade.

$\mathrm{O}$ custo diário total $(\mathrm{CDT})$ com alimentação foi menor $(\mathrm{P}<0,01)$ para os cordeiros alimentados com pó de malte de cervejaria ( $\mathrm{R} \$ 0,323)$ em relação aos cordeiros dos tratamentos com casca de soja $(\mathrm{R} \$ 0,653)$ e quirera de arroz $(\mathrm{R} \$ 0,736)$ (Tabela 7). A receita obtida por kg de peso vivo foi superior $(\mathrm{P}<0,05)$ nos tratamentos casca de soja $(\mathrm{R} \$ 63,99)$ e quirera de arroz $(\mathrm{R} \$ 77,43)$ quando comparadas com pó de malte de cervejaria ( $\mathrm{R} \$ 50,14)$. Contudo, quando se descontou da receita o custo com alimentação, a rentabilidade obtida foi superior $\mathrm{b}(\mathrm{P}<0,01)$ para o tratamento que utilizou pó de malte de cervejaria ( $\mathrm{R} \$ 1,895 / \mathrm{kg}$ de ganho de peso vivo) quando comparado aos tratamentos com casca de soja ( $\mathrm{R} \$ 0,96 / \mathrm{kg}$ de ganho de peso vivo) e quirera de arroz ( $\mathrm{R} \$ 1,155 / \mathrm{kg}$ de ganho de peso vivo). Esses resultados são uma consequência direta da redução do custo total do concentrado devido ao menor valor comercial do pó de malte de cervejaria em relação aos outros resíduos avaliados. Isso 
demonstra que a utilização de resíduos agroindustriais obtidos a baixo custo e que não comprometam acentuadamente o desempenho animal pode ser importante alternativa para terminação de cordeiros em confinamento.

Tabela 7: Valores médios para análise econômica da alimentação oferecida no experimento, de acordo com os tratamentos

\begin{tabular}{|c|c|c|c|c|}
\hline \multicolumn{3}{|c|}{ Tratamento } & \multirow[b]{2}{*}{ Média } & \multirow[b]{2}{*}{$\operatorname{Pr}>\mathbf{F}$} \\
\hline Casca de soja & Quirera de arroz & $\begin{array}{c}\text { Pó de malte de } \\
\text { cervejaria }\end{array}$ & & \\
\hline \multicolumn{5}{|c|}{ Fornecido de matéria natural de feno (kg/dia) } \\
\hline $0,578 \pm 0,08$ & $0,635 \pm 0,09$ & $0,558 \pm 0,05$ & 0,590 & 0,3919 \\
\hline \multicolumn{5}{|c|}{ Fornecido de matéria natural de concentrado (kg/dia) } \\
\hline $0,861 \pm 0,12$ & $0,945 \pm 0,14$ & $0,830 \pm 0,07$ & 0,88 & 0,4146 \\
\hline \multicolumn{5}{|c|}{ Custo diário de feno (R\$/animal/dia) } \\
\hline $0,231 \pm 0,03$ & $0,254 \pm 0,04$ & $0,223 \pm 0,02$ & 0,236 & 0,3899 \\
\hline \multicolumn{5}{|c|}{ Custo diário de concentrado (R\$/animal/dia) } \\
\hline $0,422 \pm 0,06^{\mathrm{a}}$ & $0,481 \pm 0,07^{\mathrm{a}}$ & $0,100 \pm 0,009^{b}$ & 0,334 & 0,0001 \\
\hline \multicolumn{5}{|c|}{ Custo diário total (R\$/animal/dia) } \\
\hline $0,653 \pm 0,09^{\mathrm{a}}$ & $0,736 \pm 0,11^{\mathrm{a}}$ & $0,323 \pm 0,03^{b}$ & 0,571 & 0,0002 \\
\hline \multicolumn{5}{|c|}{ Custo total de produção (R $\$$ animal) } \\
\hline $45,75 \pm 6,39^{\mathrm{a}}$ & $51,53 \pm 7,96^{\mathrm{a}}$ & $22,62 \pm 2,10^{\mathrm{b}}$ & 39,97 & 0,0002 \\
\hline \multicolumn{5}{|c|}{ Receita do peso vivo (RS/animal) } \\
\hline $143,13 \pm 13,70$ & $154,57 \pm 16,35$ & $129,15 \pm 13,77$ & 142,28 & 0,0995 \\
\hline \multicolumn{5}{|c|}{ Rentabilidade do peso vivo ( $\mathrm{R} \$$ /animal) } \\
\hline $97,37 \pm 7,59$ & $103,03 \pm 12,04$ & $106,54 \pm 12,25$ & 102,31 & 0,5096 \\
\hline \multicolumn{5}{|c|}{ Receita do ganho de peso vivo (R\$/animal) } \\
\hline $63,99 \pm 13,19^{\mathrm{a}}$ & $77,43 \pm 7,81^{\mathrm{a}}$ & $50,14 \pm 7,06^{\mathrm{b}}$ & 63,85 & 0,0107 \\
\hline \multicolumn{5}{|c|}{ Rentabilidade do ganho de peso vivo (RS/animal) } \\
\hline $18,24 \pm 9,29$ & $25,89 \pm 10,88$ & $27,52 \pm 7,10$ & 23,88 & 0,3576 \\
\hline \multicolumn{5}{|c|}{ Rentabilidade obtida por kg de ganho de peso vivo $(\mathrm{R} \$ / \mathrm{kg})$} \\
\hline $0,96 \pm 0,32^{b}$ & $1,15 \pm 0,41^{\mathrm{b}}$ & $1,89 \pm 0,29^{a}$ & 1,34 & 0,0093 \\
\hline \multicolumn{5}{|c|}{ Rentabilidade diária obtida por animal ( $\mathrm{R} \$ /$ dia) } \\
\hline $0,260 \pm 0,13$ & $0,367 \pm 0,15$ & $0,392 \pm 0,10$ & 0,34 & 0,3629 \\
\hline
\end{tabular}

Médias seguidas de letras diferentes na linha diferem $(\mathrm{P}<0,05)$ pelo teste Tukey.

\section{Conclusão}

Cordeiros alimentados em confinamento com dietas contendo quirera de arroz apresentam ganho de peso diário superior quando comparados a cordeiros alimentados com dietas contendo pó de malte de cervejaria, porém não diferindo em relação àqueles alimentados com dietas contendo casca de soja. A utilização de pó de malte de cervejaria proporciona menor custo de alimentação e um melhor resultado econômico quando comparado com a casca de soja ou quirera de arroz, mostrando ser uma boa alternativa para terminação de cordeiros em sistema de confinamento. 


\section{Referências}

1. Carvalho S, Pires CC, Macari S, Lopes JF, Moro AB, Venturini RS, Lins A, Teixeira RS. Características produtivas de cordeiros terminados em confinamento com dietas contendo diferentes teores de borra de soja. Arquivo Brasileiro de Medicina Veterinária e Zootecnia. 2014; 66(1): 259-267.

2. Amaral RM, Macedo FAF, Alcalde CR, Lino DA, Bánkuti FI, Macedo FG, Dias FB, Gualda TP. Desempenho produtivo e econômico de cordeiros confinados abatidos com três espessuras de gordura. Revista Brasileira de Saúde e Produção Animal. 2011; 12(1): 155-165.

3. Brochier MA, Carvalho S. Consumo, ganho de peso e análise econômica da terminação de cordeiros em confinamento com dietas contendo diferentes proporções de resíduo úmido de cervejaria. Arquivo Brasileiro de Medicina Veterinária e Zootecnia. 2008; 60(5): 1205-1212.

4. NRC. National Research Council. Nutrient requirements of small ruminants: sheep, goats, cervids and newworld camelids. Washington: National Academy Press, 2007. 362p. English. www.nap.edu.

5. Silva DJ, Queiroz AC. Análise de alimentos: métodos químicos e biológicos. 3.ed. Viçosa: Editora UFV, 2002. 235p. Portuguese.

6. Carvalho S, Pires CC, Wommer TP, Pelegrin ACRS, Moro AB, Venturini RS, Brutti DD. Características da carcaça de cordeiros alimentados com dietas contendo diferentes resíduos agroindustriais. Agrarian. 2012; 5(18): 409-416.

7. SAS Institute. Statistical Analysis System: user guide [CD-ROM]. Version 9.3. Cary (NC): SAS Institute Inc., 2012.

8. Fontenele RM, Pereira ES, Carneiro MSS, Pimentel PG, Cândido MJD, Filho JGLR. Consumo de nutrientes e comportamento ingestivo de cordeiros da raça Santa Inês alimentados com rações com diferentes níveis de energia metabolizável. Revista Brasileira de Zootecnia. 2011; 40(6): 1280-1286.

9. Correia BR, Oliveira RL, Jaeger SMPL, Bagaldo AR, Carvalho GGP, Oliveira GJC, Lima FHS, Oliveira PA. Consumo, digestibilidade e $\mathrm{pH}$ ruminal de novilhos submetidos a dietas com tortas oriundas da produção do biodiesel em substituição ao farelo de soja. Arquivo Brasileiro de Medicina Veterinária e Zootecnia. 2011; 63(2): 356-363.

10. Cardoso AR, Pires CC, Carvalho S, Galvani DB, Jochims F, Hastenpflug M, Wommer TP. Consumo de nutrientes e desempenho de cordeiros alimentados com dietas que contêm diferentes níveis de fibra em detergente neutro. Ciência Rural. 2006; 36(1): 215-221.

11. Kozloski GV, Trevisan LM, Bonnecarrère LM, Härter CJ, Fiorentini G, Galvani DB, Pires CC. Níveis de fibra em detergente neutro na dieta de cordeiros: consumo, digestibilidade e fermentação ruminal. Arquivo Brasileiro de Medicina Veterinária e Zootecnia. 2006; 58(5): 893-900.

12. Alves KS, Carvalho FFR, Véras ASC, Andrade MF, Costa RG, Batista AMV, Medeiros NA, Maior Junior RJS, Andrade DKB. Níveis de energia em dietas para ovinos Santa Inês: Desempenho. Revista Brasileira de Zootecnia. 2003; 32(6): 1937-1944.

13. Carvalho S, Dias FD, Pires CC, Brutti DD, Lopes JF, Santos D, Barcelos RD, Macari S, Wommer TP, Griebler L. Comportamento ingestivo de cordeiros Texel e Ideal alimentados com casca de soja. Archivos de Zootecnia. 2014; 63(241): 55-64.

14. Vieira BR, Obeid JA, Pereira OG, Valadares Filho SC, Carvalho IPC, Azevedo JAG. Consumo, digestibilidade dos nutrientes e parâmetros ruminais em bovinos alimentados com silage de capim-mombaça. Arquivo Brasileiro de Medicina Veterinária e Zootecnia. 2010; 62(5): 1148-1157.

15. Carvalho S, Brochier MA, Pivato J, Teixeira RC, Kieling R. Ganho de peso, características da carcaça e componentes não-carcaça de cordeiros da raça Texel terminados em diferentes sistemas alimentares. Ciência Rural. 2007; 37(3): 821-827.

16. Pilar RC, Pérez JRO, Teixeira JC, Muniz JA. Desempenho de cordeiros merino australiano e cruza Ile de France x Merino Australiano. Ciência e Agrotecnologia. 2003; 27(Ed. Esp.): 1652-1661. 